\title{
Oxytocin Responsivity During Skin-to-Skin Care and Diurnal Cortisol Predict Depression, Trauma and Bonding Scores at NICU Discharge in Parents of Preterm Infants
}

\author{
Bollen $\mathrm{B}^{1,2}$, Bernagie $\mathrm{C}^{1,2}$, Verhaeghe $\mathrm{J}^{1,3}$, Vanhole $\mathrm{C}^{1,2}$, Naulaers $\mathrm{G}^{1,2}$ \\ 'Department of Development and Regeneration, Women and Child, University of Leuven, Leuven, Belgium \\ ${ }^{2}$ Neonatology Department, University Hospitals Leuven, Leuven, Belgium \\ ${ }^{3}$ Department of Gynaecology and Obstetrics, University Hospitals Leuven, Leuven, Belgium
}

DOI: $10.14434 /$ do.v13i1.29079

\section{Aims}

Preterm birth is a potential traumatic experience for parents. Several studies show a high prevalence of depressive and posttraumatic stress symptoms in mothers of preterm infants. ${ }^{1}$ Hormonal changes in cortisol and oxytocin have both been implicated in these stress responses and also in parent-infant biobehavioral synchrony. ${ }^{2}$

We aimed to predict parental depression, posttraumatic stress and bonding at NICU discharge. We hypothesized that the physiological response of parents to skin-to-skin care (cortisol and oxytocin) would predict emotional distress and feelings of bonding. We also took into account early markers of parental distress (questionnaires postnatal week two).

\section{Methods}

Data were collected for the Resilience Study (NCT02623400): a prospective longitudinal cohort study performed in the University Hospitals Leuven. Parents ( $\mathrm{n}=105$ parental dyads) of 136 infants $(<34 \mathrm{w}$ GA and/or BW $<1500 \mathrm{~g})$ were included. Parents completed questionnaires in postnatal week 2 and in the week before discharge. Depressive symptoms (Edinburg Postnatal Depression Scale (EPDS)), acute trauma symptoms (Acute Stress Disorder Scale (ASDS)) and posttraumatic stress disorder (Impact of event Scale (IES) \& Traumatic event scale (TES)), and parental stress (PSS-NICU) were measured, both in mothers and fathers. Feelings of bonding were measured using the Postpartum Bonding Questionnaire (PBQ). Furthermore, parental saliva samples were collected to determine diurnal cortisol profile (awakening, $30 \mathrm{~min}, 4 \mathrm{~h}, 12 \mathrm{~h}$ later) as well as oxytocin and cortisol response during kangaroo care (KC, before, $20 \mathrm{~min}, 60 \mathrm{~min}$ ). Data were analyzed using multiple regression analysis.

\section{Results}

Mothers and fathers of preterm infants in our sample show high levels of emotional distress. Results show $76.5 \%$ of mothers, and $40.7 \%$ of fathers exceed clinical cut-off scores for postnatal depression. In general, these levels of emotional distress decrease during hospitalization.

Both in mothers and fathers, acute stress scores (postnatal week 2) but also diurnal salivary cortisol level (AUC) were significant predictors of parents' post-traumatic stress symptoms at discharge (mothers: $\mathrm{F}(2,74)=25.49, \mathrm{p}<0.0001, \mathrm{R} 2=0.41$; fathers: $\mathrm{F}(2,64)=19.31, \mathrm{p}<0.0001, \mathrm{R} 2=0.38)$. Interestingly, the salivary response in oxytocin level during $\mathrm{KC}$ is a significant predictor $(\mathrm{p}<0.01)$ of both depression and bonding scores at discharge in mothers: a higher increase in OT during $\mathrm{KC}$ care is associated with lower depression scores and with higher bonding scores in mothers.

\section{Conclusion}

This study finds high levels of emotional distress in both mothers and fathers of preterm infants. Acute stress scores and diurnal cortisol in postnatal week 2 predicted posttraumatic stress symptoms at discharge, both in fathers and mothers. Changes in salivary oxytocin level during $\mathrm{KC}$ predicted bonding and depression scores in mothers. Our findings emphasize the vulnerability of parents of preterm infants and draws attention to physiological responses underlying parental emotional distress. Our findings also highlight the need for specialized and individualized support for NICU parents.

\footnotetext{
References:

1. Hynan M, Mounts K, Vanderbilt D. Screening parents of high-risk infants for emotional distress: rationale and recommendations. Journal of Perinatology 2013,33(10):748.

2. Feldman R. Sensitive periods in human social development: New insights from research on oxytocin, synchrony, and high-risk parenting. Development and psychopathology 2015,27(2):369-95.
}

Developmental

\section{Observer}

A semi-annual publication of the NIDCAP Federation International @ 2020. The statements and opinions contained in this newsletter are solely those of the individual authors and contributors and not necessarily of the NIDCAP Federation International. Articles from the Developmental Observer, duly acknowledged, may be reprinted with permission. Please contact us at: developmentalobserver@nidcap.org.

Senior Editor Associate Editors

Associate Editor for Science

Column Editor
Kaye Spence AM

Diane Ballweg, APRN, CCNS, Deborah Buehler, PhD, Sandra Kosta, BA gretchen Lawhon, PhD, RN, FAAN, Maria Maestro Lopez, MD Jeffrey R. Alberts, PhD

Debra Paul OTR/L

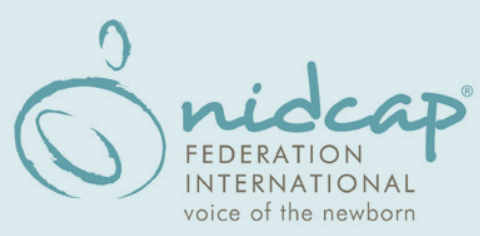

Contributions

We would like to thank all of our individual donors for their generous support of the NFI and its continuing work. 